DOI: $10.12731 / 2306-1561-2013-4-17$

\title{
DATABASE DESIGN OF MONITORING SYSTEM TECHNICAL- ECONOMIC INDICATORS OF MOTOR COMPANY
}

\author{
Ataeva S.K., Borsch V.V., Zaitsev D.V., Chugunova D.N.
}

Abstract

The monitoring system of technical and economic indicators of trucking companies need for operational decision making, which should be supported by information support functions. To implement the system designed database developed mathematical methods and models, as well as software and simulation system to automate the process of calculating the efficiency of the implementation of various strategies for enterprise development.

The results of the statistical analysis of the technical and economic performance of the enterprise "First avtokombinat G.L. Krauze".

Keywords: system monitoring, motor company, database, OLAP- technology, the relational model.

\section{УДК 681.3}

\section{ПРОЕКТИРОВАНИЕ БАЗЫ ДАННЫХ СИСТЕМЫ МОНИТОРИНГА ТЕХНИКО-ЭКОНОМИЧЕСКИХ ПОКАЗАТЕЛЕЙ АВТОТРАНСПОРТНЫХ ПРЕДПРИЯТИЙ}

\author{
Атаева С.К., Борщ В.В., Зайцев Д.В., Чугунова Д.Н.
}

\section{Аннотация}

Система мониторинга технико-экономических показателей автотранспортных предприятий необходима для оперативного принятия решений, что должно подкрепляться функциями информационной поддержки. Для реализации системы проектируется база данных, разрабатываются математические методы и модели, а также программно-моделирующий комплекс для автоматизации процессов расчета эффективности от внедрения различных стратегий развития предприятий.

Приведень результаты статистического анализа технико-экономических показателей предприятия ОАО «Первый автокомбинат им. Г.Л. Краузе».

Ключевые слова: система мониторинга, автотранспортное предприятие, база данных, ОLAР-технологии, релящионная модель. 


\section{Классификация показателей}

Для решения вопросов выбора стратегии управления используется метод $\mathrm{K}$ средних. Часто показатели кластеризации имеют различные масштабы измерений, поэтому предполагается задание степени важности каждого при разбиении на группы и последующем изменении масштаба в соответствии со значением важности. С другой стороны может быть сделан переход к иной системе показателей.

В данном случае, при разбиении на кластеры существенное влияние оказывают несколько показателей, а именно, объем перевозок, время в наряде и среднесуточный пробег. Изменяя важность в связи с контекстом решения задачи кластеризации, можно получить новые разбиения (рисунок1).

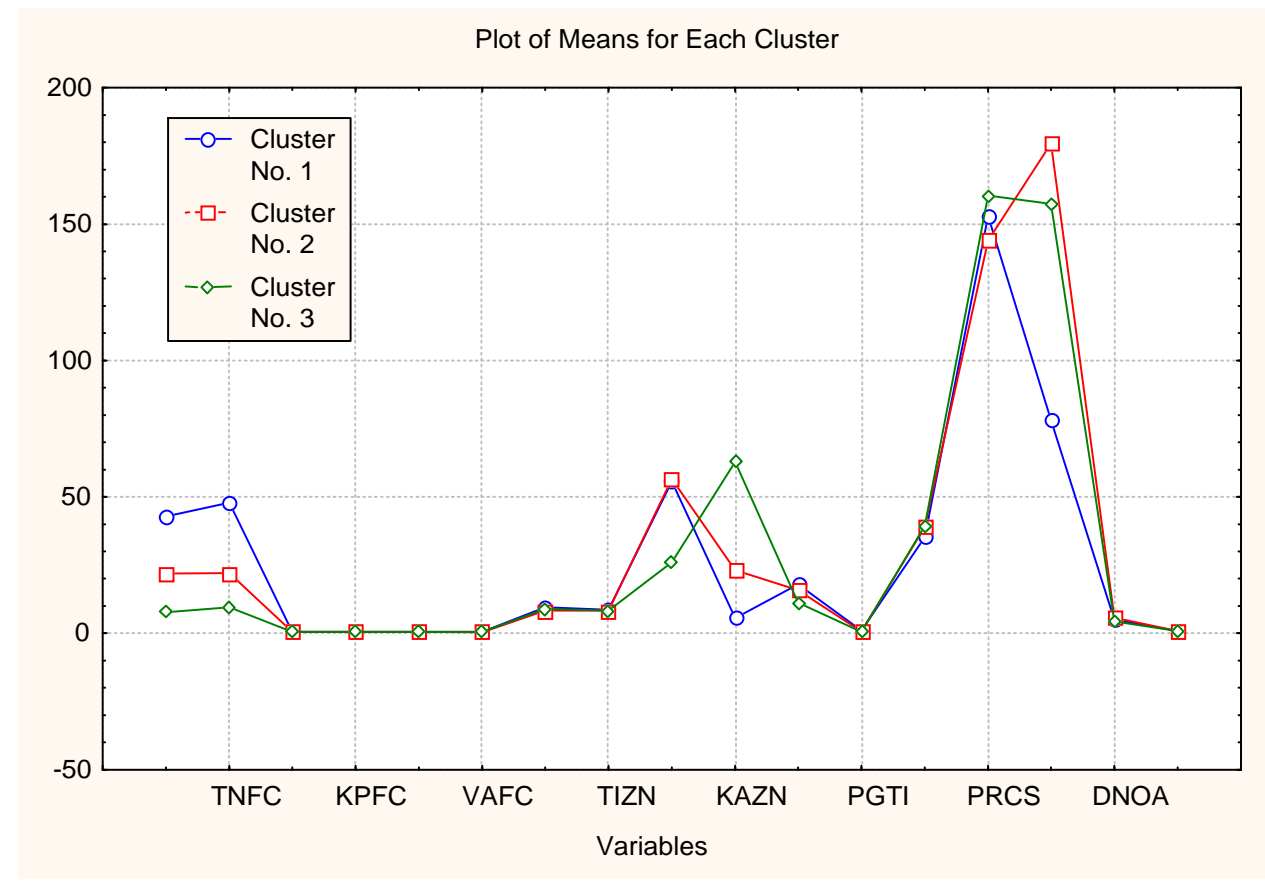

\section{Рисунок 1 - График средних значений показателей кластеров}

На основе проведенного статистического анализа технико-экономических показателей поставлена задача минимизации стоимостей перевозок сразу по всем видам транспортных средств (ТС), находящихся в распоряжении автотранспортного предприятия:

$$
\sum_{q=1}^{1 q} \sum_{a=1}^{l a} C A Q_{a q} \cdot A Q_{a q} \rightarrow \min
$$

где: $\left\|A Q_{a q}\right\|$ - искомая матрица распределения объемов; $\left\|C A Q_{i j}\right\| \mathrm{i}=1 . . \mathrm{I}_{q}, \mathrm{j}=1 . . \mathrm{I}_{\mathrm{a}}$ матрица стоимостей транспортировки единицы q-го материала а-ым типом $\mathrm{TC} ; \| \mathrm{VAQ}$ $\mathrm{i}=1 . . \mathrm{I}_{\mathrm{q}}, \mathrm{j}=1 . . \mathrm{I}_{\mathrm{a}}$ - матрица максимальных объемов транспортировки q-ым ТС за плановый период. 
В условиях такой постановки выполняется уравнение баланса по типам материалов, но баланс по возможностям транспортных средств не выполняется. Метод решения транспортной задачи позволяет использовать несбалансированные поставки. При этом определение коэффициента использования транспортных средств также представляет собой оптимизационную задачу:

$$
\forall a \quad \sum_{q=1}^{l q} \frac{A Q_{a q}}{V A Q_{a q}} \rightarrow \min
$$

Предлагается эвристический алгоритм решения данной задачи с использованием процедуры последовательного приближения, в которой на каждом шаге решается транспортная задача с уточненными ограничениями.

На основании разработанных моделей предлагается динамический алгоритм корректировки производственных планов с учетом согласования работы транспортного звена. Основой алгоритма является модель объекта, предусматривающая возможность вариации сроками отдельных этапов работ на каждом из объектов.

Каждый этап представляет собой тройку $\left(\tau \mathrm{j}, \mathrm{r}_{\mathrm{j}}, \mathrm{f}_{\mathrm{j}}\right)$, где $\tau_{\mathrm{j}}$ - длительность работы, $\mathrm{r}_{\mathrm{j}} \geq 0$ - уровень использования ресурсов в процессе выполнения этапа, $\mathrm{f}_{\mathrm{j}}=\mathrm{f}_{\mathrm{j}}(\mathrm{t}), \mathrm{t} \in[0, \mathrm{~T}]$ функция времени, определяющая затраты на реализацию этапа в момент $t$. Предполагается, что этап не прерывается и продолжается с использованием всех изначально выделенных ресурсов. Пусть $S=\left\{\mathrm{t}_{\mathrm{j}}(\mathrm{S})\right\}$ - непосредственно структура планов производства работ, тогда:

$$
g_{i}(S)=\sum_{j=1}^{N} g_{i j}\left(t_{j}(S)\right)
$$

где $\mathrm{g}_{\mathrm{j} j}\left(\mathrm{t}_{\mathrm{j}}(\mathrm{S})\right)=\mathrm{r}_{\mathrm{j}} \mid \Delta \mathrm{i} \cap\left[\mathrm{t}_{\mathrm{j}}(\mathrm{S})-\tau_{\mathrm{j}}, \mathrm{t}_{\mathrm{j}}(\mathrm{S})\right]$ - объем использования ресурсов на интервале $\Delta i$ в процессе выполнения ј-го этапа. Допустимые общие объемы использования ресурсов на каждом интервале $\Delta \mathrm{i}$ определяются заданными уровнями $\mathrm{g}_{0 \mathrm{i}}, \mathrm{i}=1 . . \mathrm{m}$.

Задача оптимизации состоит в поиске вектора $\mathrm{S}=\left\{\mathrm{t}_{\mathrm{j}}(\mathrm{S})\right\}$, доставляющего минимум затрат при ограничениях на объемы транспортировок на каждом интервале, что формально записывается:

$$
\begin{gathered}
G_{0}(x)=\sum_{j=1}^{N} g_{o j}\left(x_{j}\right) \rightarrow \boldsymbol{m i n} \\
G_{i}(x)=\sum_{j=1}^{N} g_{i j}\left(x_{j}\right) \leq g^{0}, \quad i=1 . . m
\end{gathered}
$$

В результате решения задачи получается план выполнения всего комплекса работ, который сбалансирован как относительно ритмичности работы на отдельных производственных участках, так и относительно ритмичности транспортного звена. 
Полученный план можно считать опорным планом для его дальнейшего анализа с учетом динамики условий функционирования.

\section{Многомерное представление данных}

Весь спектр задач статистического анализа технико-экономических показателей автотранспортного предприятия и последующее решение задач оптимизации использования транспортных средств, ставит задачу формализованного описания исходных, результирующих и модельных данных.

Однако для решения поставленных задач необходима структуризация данных, которая обычно отсутствует на предприятиях. Данные, в основном, хранятся в простых таблицах, что не дает возможности использования OLAP-технологий для оперативного анализа. В связи с этим предлагается преобразование системы таблиц в базы данных, которые необходимы для использования разработанной программы визуализации данных.

Показанная на рисунке 2 (в случае с тремя простыми измерениями) структура многомерного куба (стрелки показаны направления агрегации) включает в себя показатель: количество продукции и три измерения:

- менеджеры по продажам (ось оМ),

- модели транспортных средств (ТС) (ось оС),

- временное измерение с единицей “месяц” (ось оТ).

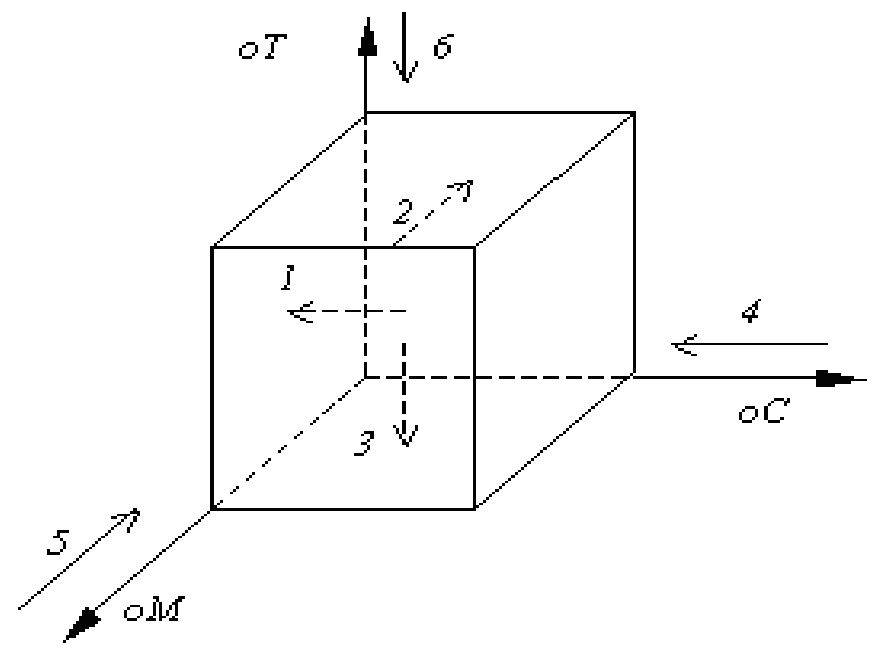

\section{Рисунок 2 - Трёхмерный OALP-куб с простыми измерениями}

Обозначим С, М, Т - множества членов соответствующих измерений “модели TC”, “менеджеры”, “месяцы”. Обозначим также количество членов в каждом из измерений $\mathrm{nc}=|\mathrm{C}|, \mathrm{nm}=|\mathrm{M}|$, $\mathrm{nt}=|\mathrm{T}|$. Члены этих измерений будем обозначать соответственно $\mathrm{mc}, \mathrm{mm}, \mathrm{mt}$.

Для получения агрегированных значений в разрезе менеджеров и месяцев требуется просуммировать первоначальные значения показателей по всем моделям для 
каждой комбинации $(\mathrm{mt}, \mathrm{mm})$. На рисунке 2 направление агрегирования обозначено стрелкой 1. Количество агрегированных таким образом значений равно nmnt. Агрегированные таким образом значения показателей располагаются на плоскости (оМ, oT).

Аналогично получим число агрегатов (ncnt) для всех комбинаций (mc, mt) при суммировании показателей по всем членам измерения “менеджеры”. Количество агрегатов для всех комбинаций $(\mathrm{mc}, \mathrm{mm})$ при агрегации по временному измерению равно $\mathrm{nm}$ nc. Число агрегатов в разрезе членов одного из измерений равно числу членом соответствующего измерения nm, nc и $\mathrm{nt}$.

\section{Схема базы данных}

Несмотря на то, что большинство объектов не предполагается детализировать на ранних этапах разработки, такие объекты, как модели данных табличного вида (ДТВ) и модели реляционной базы данных (РБД) рассмотрены детально. Они являются основными компонентами моделей ДТВ и РБД, и именно отличия этих моделей в основном определяет состав необходимых методов решения задач проектирования БД.

Схематичное представление модели РБД приведено на рисунке 3

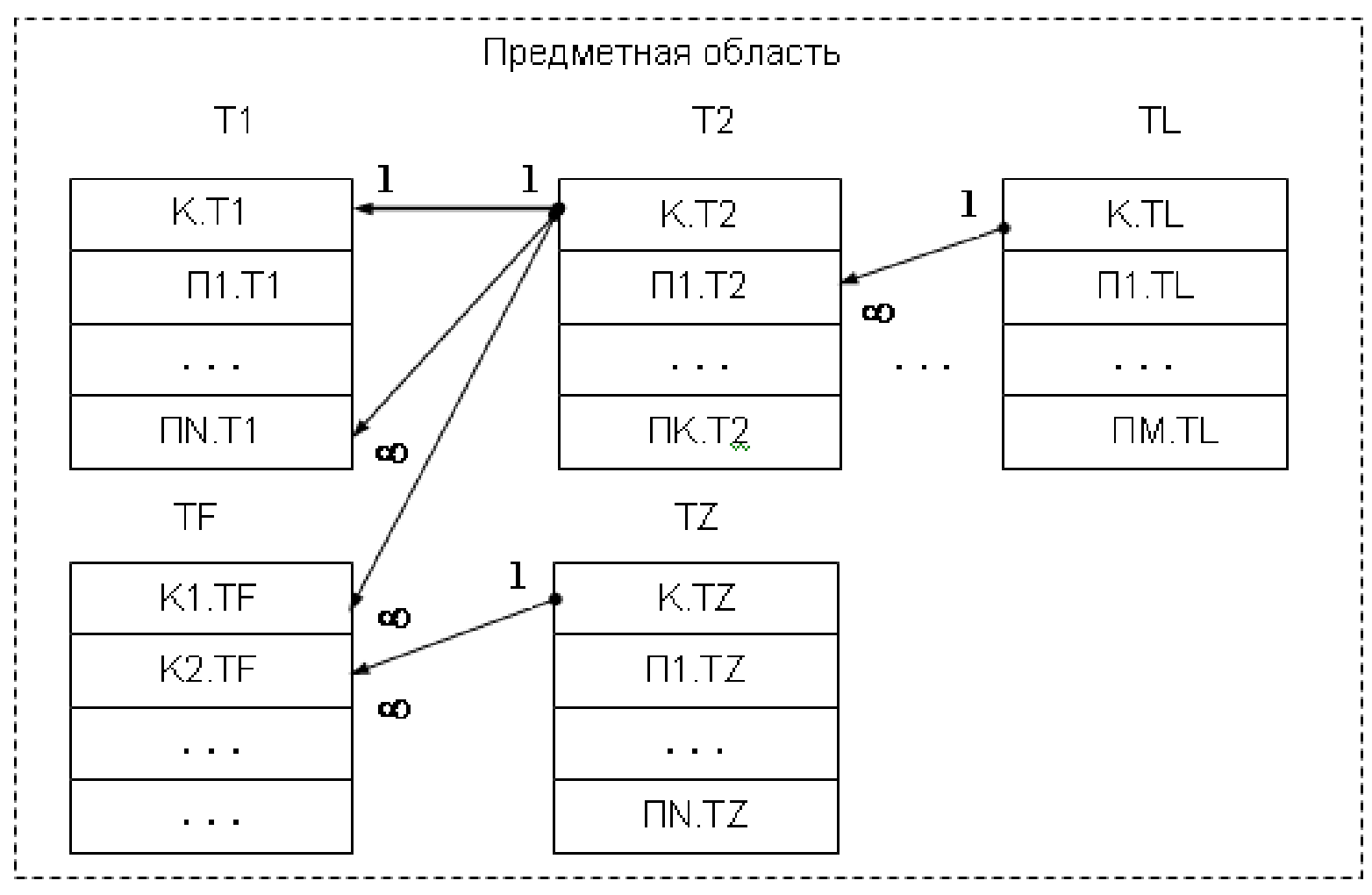

Рисунок 3 - Схематичное представление РБД

Представление модели ДТВ - на рисунке 4. 


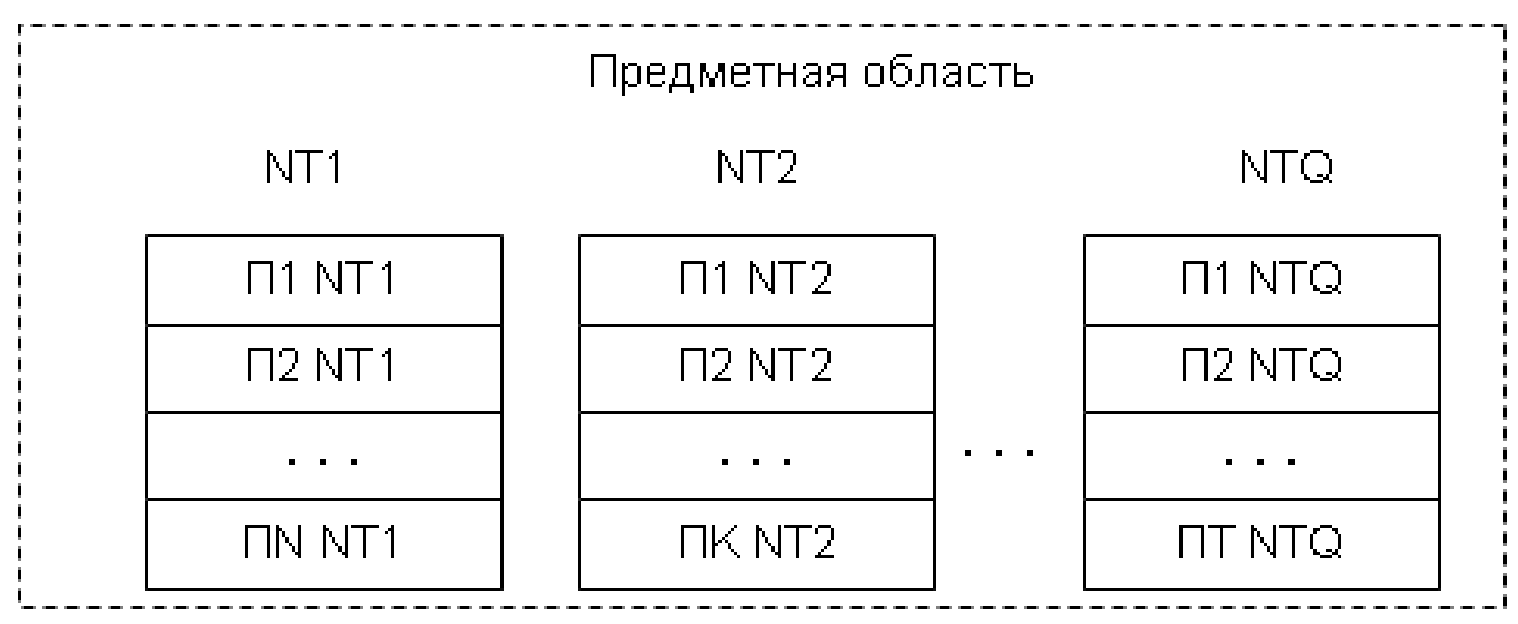

\section{Рисунок 4 - Схема данных табличного вида}

Данные табличного вида представляются множеством DT $=\{\mathrm{Z}, \mathrm{D}\}, \quad$ где $\mathrm{Z}-$ множество заголовков, D - множество данных.

$$
\mathrm{Z}=\left\{\mathrm{Z}_{1}, \ldots, \mathrm{Z}_{2}, \ldots, \mathrm{Z}_{\mathrm{n}}\right\}, i=\overline{1, n} ; \mathrm{n}>=1,
$$

где $\mathrm{n}$ - степень множества заголовков.

Допустима ситуация, когда $\mathrm{Z}_{\mathrm{i}}=\mathrm{Z}_{\mathrm{m}}, i=\overline{1, n}, m=\overline{1, n} ; \mathrm{i} \neq \mathrm{m}$,

где $\mathrm{n}$ - степень множества заголовков, то есть, полное совпадение заголовков.

В данных табличного вида возможны подзаголовки 1-го уровня, что формально выглядит следующим образом.

$$
\mathrm{Zi}=\left\{\mathrm{PZ}_{\mathrm{i} 1}, \ldots, \mathrm{PZ}_{\mathrm{ij}}, \ldots, \mathrm{PZ}_{\mathrm{ik}}\right\}, j=\overline{1, k} ; \mathrm{k}>=2,
$$

где $\mathrm{k}$ - степень множества подзаголовков і-го заголовка.

$$
\mathrm{Z} \mathrm{p}=\left\{\mathrm{PZ} \mathrm{p}_{1}, \ldots, \mathrm{PZ}_{\mathrm{pt}}, \ldots, \mathrm{PZ}_{\mathrm{pm}}\right\}, t=\overline{1, m} ; \mathrm{m}>=2,
$$

где $\mathrm{m}$ - степень множества подзаголовков р-го заголовка.

Допустима ситуация, когда:

$$
\mathrm{PZ}_{\mathrm{ij}}=\mathrm{PZ} \text { pt. }
$$

В данных табличного вида возможны подзаголовки 2-го уровня, что формально выглядит следующим образом.

$$
\mathrm{PZ}_{\mathrm{ij}}=\left\{\mathrm{PPZ} \mathrm{Z}_{\mathrm{ij} 1}, \ldots, \mathrm{PPZ} \mathrm{Z}_{\mathrm{ijm}}, \ldots, \mathrm{PPZ} \mathrm{Z}_{\mathrm{ijf}}\right\}, m=\overline{1, f} ; \mathrm{f}>=2,
$$

где $\mathrm{f}$ - степень множества подзаголовков 2-го уровня іј-го подзаголовка 1-го уровня.

$$
\mathrm{PZ}_{\mathrm{pt}}=\left\{\mathrm{PPZ} \mathrm{pt}_{1}, \ldots, \mathrm{PPZ}_{\mathrm{ptr}}, \ldots, \mathrm{PPZ}_{\mathrm{ptg}}\right\}, r=\overline{1, q} ; \mathrm{q}>=2,
$$

где q - степень множества подзаголовков 2-го уровня pt-го подзаголовка 1-го уровня. 
Допустима ситуация, когда:

$$
\begin{gathered}
\mathrm{PPZ}_{\mathrm{ijm}}=\mathrm{PPZ} \mathrm{ptm}_{\mathrm{pt}} . \\
\mathrm{D}=\{\mathrm{SD}, \mathrm{Z}\},
\end{gathered}
$$

где SD - множество строк данных.

\section{Метод проектирования базы данных}

Несмотря на некоторое сходство модели данных табличного вида и модели реляционной таблицы, в них имеются существенные различия. В результате реализован следующий метод проектирования РБД на основе ДТВ.

Hа первом этапе (по аналогии с описанием процесса взаимодействия решающих систем), используя отличия моделей ДТВ и РБД, в операторной форме, описываются шаги преобразования ДТВ в РБД. Формируются связи между ними, определяются правила и порядок их использования. Такое описание разработано с целью выявления основных компонент разрабатываемой интерактивной системы выявления основных связей между ними, построения модели процесса. Под оператором согласно его определению понимается отображение ОР: $\mathrm{X} \rightarrow \mathrm{Y}$, в котором множества X и Y являются множествами функций с элементами $\mathrm{x}(\mathrm{t})$ и $\mathrm{y}(\mathrm{t})$. Формально факт преобразования функции $\mathrm{x}(\mathrm{t})$ в функцию $\mathrm{y}(\mathrm{t})$ посредством выполнения оператора ОР отмечается следующим образом: $\mathrm{y}(\mathrm{t})=\mathrm{OP}(\mathrm{x}(\mathrm{t}))$.

Ha втором этапе операторная модель используется в качестве исходной формализации для разработки модели процесса проектирования и формируется соответствующая сеть.

Ha mpeтьем эmane выявляются и исключаются дефекты модели, а, следовательно, исключаются дефекты объекта моделирования. В конечном итоге строится сетевая модель процесса проектирования, свободная от концептуальных ошибок.

Ha четвертом этапе с помощью деревьев достижимости анализируются динамические свойства процесса проектирования.

Для последовательного развертывания операторной модели в работе рассмотрены все возможные сочетания пунктов несоответствия модели ДТВ и модели РБД: ДТВ не реляционные таблицы; ДТВ - ненормализованные таблицы; в ДТВ отсутствуют ключевые поля; таблицы ДТВ не связаны между собой. Соответствующие постфиксы в операторах модели “p”, “н”, “к”, “c”. Индекс “и” используется для оператора импортирования, который задействован при любом сочетании.

В результате таких преобразований появляется возможность оперативного отображения данных в системе мониторинга. 


\section{Список информационных источников}

[1] Гоголин С.С., Подпорин Д.И. Планирование доставки грузов с использованием информационно-аналитической системы. / Современные технологии управления в автотранспортных системах: сб. науч. тр. Ф-та «Управления» МАДИ(ГТУ). - М.: МАДИ, 2007. - С.57-63.

[2] Гоголин С.С. Мониторинг и управление технологическими процессами на производственных предприятиях с распределенной структурой. / Аналитикоимитационное моделирование и ситуационное управление в промышленности, строительстве и образовании. Сб. науч. тр. МАДИ(ГТУ). - М.: МАДИ, 2008. - С. $24-30$.

[3] Губанов А.И., Ефименко Д.Б., Николаев А.Б., Остроух А.В. Концепция автоматизированной навигационной системы диспетчерского контроля и учета работы транспорта нефтедобывающих и нефтеперерабатывающих предприятий // Автоматизация, телемеханизация и связь в нефтяной промышленности. - М.: ВНИИОЭНГ, 2011. - № 11 - С. 12- 4.

[4] Бернер Л.И., Никаноров В.В., Николаев А.Б., Рощин А.В. Системы поддержки принятия диспетчерских решений в многоуровневых автоматизированных системах управления технологическими процессами добычи нефти и газа. // Автоматизация, телемеханизация и связь в нефтяной промышленности. - М.: ВНИИОЭНГ, 2013. - №1. - С. 59-68.

[5] Остроух, А.В. Информационные технологии в научной и производственной деятельности [Текст] / [ред. А.В. Остроух] - М: ООО "Техполиграфцентр", 2011. 240 с. - ISBN 978-5-94385-056-1.

[6] Польгун М.Б. Автоматизация процессов диспетчерского управления городским пассажирским транспортом. / Д.Б. Ефименко, А.Б. Николаев, А.В. Остроух, М.Б. Польгун / Промышленные АСУ и контроллеры. - М.: «Научтехлитиздат» - №5. 2013. - C. 10-16.

[7] Тянь Юань. Разработка информационно-аналитической системы мониторинга технологических процессов предприятия автомобильной промышленности / А.В. Остроух, Юань Тянь // В мире научных открытий. - Красноярск: «Научноинновационный центр», 2013. - № 8.2 (44). - С. 191-205.

[8] Тянь Юань. Оперативный мониторинг внутренней среды промышленных предприятий // Автоматизация и управление в технических системах. - 2012. - № 1; URL: auts.esrae.ru/1-33 (дата обращения: 24.09.2013).

[9] Тянь Юань. Современные методы и подходы к построению систем управления производственно-технологической деятельностью промышленных предприятий / А.В. Остроух, Юань Тянь // Автоматизация и управление в технических системах. - 2013. - № 1(3); URL: auts.esrae.ru/3-53 (дата обращения: 24.09.2013).

[10] Тянь Юань. Мониторинг процесса производства сухих строительных смесей / А.В. Остроух, Вэй Пьо Аунг, Юань Тянь // Наука и образование в XXI веке: Теоретические и прикладные вопросы науки и образования: сб. науч. тр. по матлам Междунар. науч. - практ. конф. 30 сентября 2013 г.: Часть 1. - Тамбов: ТРОО «Бизнес-Наука-Общество», 2013. - С. 138-140. 
[11] Тянь Юань. Мониторинг технологического процесса производства керамического кирпича / А.В. Остроух, Р.P. Чаудхари, Юань Тянь // Наука и образование в XXI веке: Теоретические и прикладные вопросы науки и образования: сб. науч. тр. по мат-лам Междунар. науч. - практ. конф. 30 сентября 2013 г.: Часть 1. - Тамбов: ТРОО «Бизнес-Наука-Общество», 2013. - С. 140-143.

[12] Тянь Юань. Интеграция компонентов системы мониторинга /А.В. Остроух, Юань Тянь // Молодой ученый. - Чита: ООО «Издательство Молодой ученый», 2013. №10. - С. 182-185. 